\title{
A Novel and Collaborative Network Channel Coded Mobile Communication Architecture
}

\author{
Marium Jalal Chaudhry ${ }^{(1,2)}$, Sandeep Narayanan ${ }^{(1,2)}$, Marco Di Renzo ${ }^{(3)}$, Fabio Graziosi ${ }^{(1,2)}$, Azhar Ul Haq $^{(2)}$ \\ (1) WEST Aquila s.r.l., Via G. Gronchi 18, Nucleo Industriale di Pile, 67100 L’Aquila, Italy \\ (2) University of L'Aquila, Center of Excellence for Research DEWS, Via G. Gronchi 18, Nucleo Industriale di Pile, 67100 L'Aquila, Italy \\ (3) Laboratory of Signals and Systems (L2S), UMR 8506 CNRS - SUPELEC - Univ Paris-Sud, 3 rue Joliot-Curie, 91192 Gif-sur-Yvette (Paris), France \\ E-Mail: marium.jalal@westaquila.com, marco.direnzo@1ss.supelec.fr, fabio.graziosi@univaq.it
}

\begin{abstract}
This paper presents a novel algebraic framework of collaborative network channel coding scheme in a network with multiple-access relay channel. The proposed scheme helps reduce the computational complexity while enhancing energy efficiency of the wireless network. The devised scheme is intended for practical scenarios where channels exhibit various fading conditions over all the wireless links. We use network coding at the relay to give diversity boost at the receiver with lesser number of transmitted signals and lesser energy consumption. The setup demonstrates improved performance that is instrumental for the effective use of joint network channel coding (JNCC) for practical wireless networks. We further present a performance metric Energy Efficiency for the better performance evaluation.

Index Terms-Network-Coded Cooperative Diversity, Channel Coding, Energy Efficiency, Error Probability, Performance Analysis.
\end{abstract}

\section{INTRODUCTION}

The invention of joint Network-Channel coding for the design of energy efficient wireless systems have shown significant improvements being extensively used in corporative relay channel to obtain improved diversity gains [1]. NetworkCoded Cooperative Diversity (NCCD) schemes are one of the major protocols that combine relay-aided Cooperative Diversity (CD) and Network Coding (NC). Besides various overwhelming applications and advantages of $\mathrm{NC}$ over conventional routing techniques, there are some problems as well like; a primary trouble is that $\mathrm{NC}$ takes into account the error propagation of wireless networks in which loss is high due to various fading conditions and corrupted packets get combined by some intermediate nodes and may propagate through the network towards destination. It might render impossible to decode the original packets. Basically channel fading is one of the major underlying causes of performance degradation and energy consumption in wireless networks [2]-[4]. Mathematical frameworks, analysis and results for error performance and diversity analysis along with network code design are still unavailable for arbitrary networks. Some mathematical frameworks for the analysis of NCCD protocols have been proposed in literature [5],[6]. The complexity of several general network coding problems has been analyzed in [7]-[13]. But, they are applicable to either simple network topologies or to specific types of network codes and modulation types [2],[3]. We shall study the benefits of collaborative networkchannel coding scheme for a canonical two source two relay multiple access channels with the block fading channels. An example can be a cellular mobile communication system where two mobile stations cooperativelv send information to beneficial in modelling with slowly varying fading channels, where duration of a block-fading period is determined by the channel coherence time. In particular we intend to study the benefit of network coding in the diversity gain in the presence of AWGN noise and block fading channel. We use expander codes for channel codes to make polynomial time and simple coding and encoding possible. Expander codes are a variation of random linear network codes with low computational complexities in terms of encoding and decoding efficiencies. Against this background, our contribution is four fold: i) the optimal demodulator at the destination, which takes into account demodulation errors at the relays, is developed based on collaboration of network coding and expander codes ii) the diversities are studied analytically, and second-order diversity is proved; iii) simulations are used to show that our proposal outperforms other cooperative or collaborative protocols; iv) Above 90\% Energy Efficiency has been achieved and with less error probabilities.

The remainder of this paper is organized as follows. The first Subsection describes the proposed algorithm, Section II is about system model. In Section III Broadcasting, Network Coding and Detection phases are explained in detail, the Section IV is about performance analysis of optimal demodulator and its achievable diversity. The last Section presents the numerical results to substantiate our claims. Finally Section $\mathrm{V}$ concludes the paper.

\section{A. Proposed Algorithm}

The algorithm suggested is composed of two phases. In phase I we find $\mathrm{h}$ edge disjoint paths from source $\mathrm{s}$ to each destination. In phase II collaborative network and expander coding will be performed on broadcasted signals

We focus on a basic time-division multiple access relay channel (TD-MARC) for wireless network consisting of two sources, two relays and one destination as shown in Figure 1. In first time slot source $s_{1}$ sends a message $A$ to both relays, and destination. In second time slot source $s_{2}$ sends a message $B$ to both relays, and destination. Both relays perform network coding on the signals received from both sources. Relay $r_{1}$, and $r_{2}$ send the network coded message $\alpha_{1} A+\beta_{1} B$, $\alpha_{2} A+\beta_{2} B$ in third and fourth time slots respectively. Here $\alpha_{1}, \alpha_{2}, \beta_{1}, \beta_{2} \in G F(q)$ such that $\alpha_{1} A+\beta_{1} B, \alpha_{2} A+\beta_{2} B$ are linearly independent. In other words two redundant signals are sent i.e., in stead of receiving two signals, the destination receives four signals which in turn boosts the diversity gain. In case the channel condition is not perfect or ideal an error or failed transmission over one of the channels can be compensated by the extra copies of the signal received. 


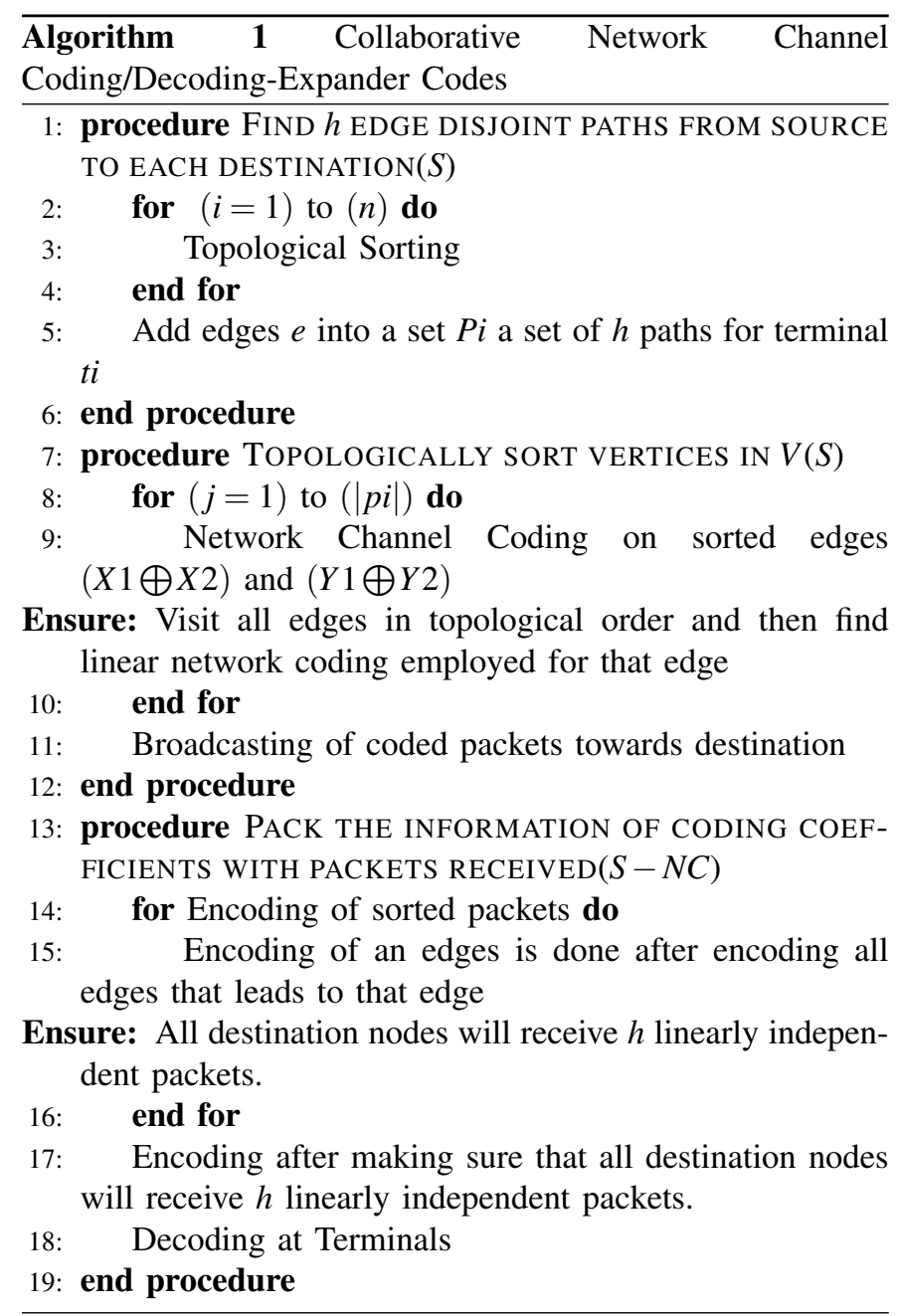

This is required in particular because the practical channel exhibit fading and noise. In this work, for the above mentioned network we consider that both the sources modulate the signal to be transmitted after encoding them using linear block code. At the relay only the demodulation of the received signal is done before applying network code. The motivation here is two folded; 1) It shall result in simpler and lesser computations at the relay nodes, 2) The two code words received from the two sources shall be mixed in a way to make another valid codeword with the errors being added dues to the assumption of a linear channel model that will eliminate the need of error detecting or correcting at the relays altogether. In particular, to collaboratively perform source, network coding works in a distributed manner among the all nodes of a generic cooperative and multihop network.

Using the proposed scheme we have devised an energy efficient collaborative network channel codes for Time-division Multiple Access Relay Channels with error-correcting capabilities for noisy and fading channels using a new class of expander codes [18]. This special type of linear block code are constructed by using bipartite expander graphs. In fact these are the only known linear block codes that can be both encoded and decoded in time proportional to the block length of the code in polynomial time. In such a system much of the energy is consumed for re-transmitting the packets with errors, our goal of combining the error correcting capabilities of block

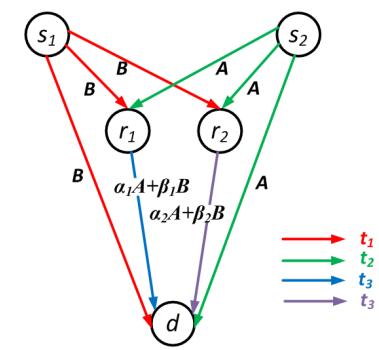

Fig. 1. A canonical two sources, two relays and one destination wireless network. In first time slot source $s_{1}$ sends a message $A$ to both relays, and destination. In second time slot source $s_{2}$ sends a message $B$ to both relays, and destination. Both relays perform network coding on the signals received from both sources. Relay $r_{1}$, and $r_{2}$ sends the network coded message $\alpha_{1} A+\beta_{1} B$, $\alpha_{2} A+\beta_{2} B$ in thirds and fourth time slots respectively.

codes with the network codes result in an energy efficient high throughput scheme. Furthermore we shall measure the Energy Efficiency (EE) defined as a parameter that gives the advantage in terms of energy for using proposed coding over conventional forwarding to achieve the same dimension for the diversity. We present the Average Symbol Error Probability (ASEP) performance as a function of the average transmit energy per coded symbol $E_{C_{i}}$. A comparison with two state-of-the-art schemes has also been presented and RAER (Relative Average Energy Ratio per transmitted symbol) has been calculated.

\section{SyStem ModeL}

Consider the multiple-access two source two relay model shown in Figure 1 consisting of two sources $s_{1}$ and $s_{2}$, two relay nodes $r_{1}, r_{2}$ and one destination node $d$. We assume that each node is communicating with a single antenna in a halfduplex environment. System block diagram for source $s_{i}$, and relay $r_{i}$, for $i \in\{1,2\}$ is shown in Fig 2 and Fig 3 respectively. All the channel links are assumed to be block fading channels and are subjected to complex Additive White Gaussian Noise (AWGN). Further, we define the following symbols:

- Fading coefficient for channel between source $s_{i}$ and relay $r_{j}: h_{s_{i}, r_{j}}$, where $i, j \in\{1,2\}$

- Fading coefficient for channel between source $s_{i}$ and destination $D: h_{s_{i}, d}$, where $i \in\{1,2\}$

- Fading coefficient for channel between relay $r_{i}$ and destination $D: h_{r_{i}, d}$, where $i \in\{1,2\}$

- Fading coefficient for any channel is assumed to be for block fading.

- Noise sample sequence for channel between source $s_{i}$ and relay $r_{j}: n_{s_{i}, r_{j}}$, where $i, j \in\{1,2\}$

- Noise sample sequence for channel between source $s_{i}$ and destination $d: n_{s_{i}, d}$, where $i \in\{1,2\}$

- Noise sample sequence for channel between relay $r_{i}$ and destination $d: n_{r_{i}, d}$, where $i \in\{1,2\}$

- Noise sample sequence for any channel is comprised of a zero-mean complex Gaussian random variable with variance $N_{0}$

- Input data streams to source $s_{i}$ for time slot $t: X_{i}(t)=$ $\left\{x_{i}^{1}(t), x_{i}^{2}(t), \cdots, x_{i}^{N_{i}}(t)\right\}$, where $i \in\{1,2\}$ and $N_{i}=N \forall i$. Note that without loss of generality any difference in the length of the input bits can be compensated by padding zeros at the end. 
- Output of the $(M, N, d)_{2}$-Expander Codes at relay $r$ for time slot $t: C_{r}(t)=\left\{c_{r}^{1}(t), c_{r}^{2}(t), \cdots, c_{i}^{M}(t)\right\}$

- Output of L-QAM modulator for time slot $t$ at relay $r_{i}$ : $Y_{r_{i}}(t)$. Without loss of generality we assume that $M$ is multiple of $L$.

We further assume an ideal synchronization and channel estimation at the receiver side. Receiver shall first demodulate the three signals received on its three incoming channels and then shall decode the symbols using decoder. Next, it shall decode the two original signals sent out by combining the signals received.

\section{Broadcasting, Network Coding and Detection}

In this section we explain the broadcasting, network coding and detection phases of proposed scheme step by step. At time slot $t_{1}$ the source $s_{1}$ shall first channel code the input data $X_{1}\left(t_{1}\right)$ stream by using expander codes and then modulate it. The modulated signal sent by source $s_{i}$ is:

$$
Y_{i}\left(t_{i}\right)=\exp \left(\frac{j 2 \pi}{L} C_{i}\right) \sqrt{E_{C_{i}}}
$$

Here $E_{C_{i}}$ is the symbol energy transmitted is:

$$
E_{C_{i}}=\log _{2}(Q) * S N R * N_{0}
$$

Where $N_{0}$ is noise power spectral density. The received signals at the relay $r_{j}$ from source $s_{i}$ is:

$$
Y_{r_{j}}^{i} \hat{\left(t_{i}\right)}=Y_{i}\left(t_{i}\right) h_{s_{i}, r_{j}}+n_{s_{i}, r_{j}}
$$

Similarly the received signals at the destination $d$ from source $s_{i}$ is:

$$
\left.Y_{d}^{s_{i}} \hat{(} t_{i}\right)=Y_{i}\left(t_{1}\right) h_{s_{i}, d}+n_{s_{i}, d}
$$

Furthermore the received signals at the destination $d$ from source $r_{i}$ is:

$$
Y_{d}^{r_{i}}\left(\hat{t}_{2+i}\right)=Y_{r_{i}}\left(t_{2+i}\right) h_{r_{i}, d}+n_{r_{i}, d}
$$

Here $Y_{r_{i}}$ is the modulated signal of the input $\alpha_{i} X_{r_{i}}^{1}(t)+$ $\beta_{i} X_{r_{i}}^{2}(t)$ at the relay $r_{i}$ is given by:

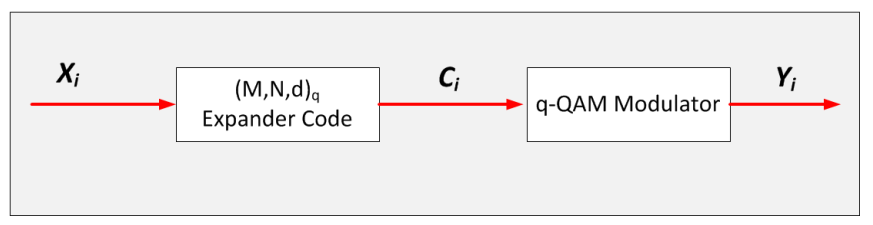

Fig. 2. Block diagram for source $s_{i}$, where $i \in\{1,2\}$.

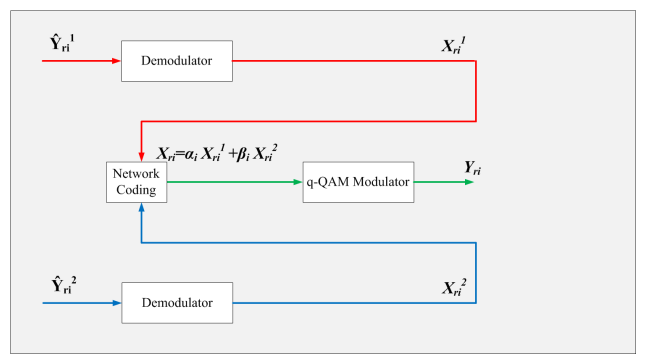

Fig. 3. Block diagram for relay $r_{i}$, where $i \in\{1,2\}$.
$Y_{r_{i}}\left(t_{2+i}\right)=\exp \left(\frac{j 2 \pi}{L} \alpha_{i} X_{r_{i}}^{1}(t)+\beta_{i} X_{r_{i}}^{2}(t)\right) \sqrt{E_{\alpha_{i} X_{r_{i}}^{1}(t)+\beta_{i} X_{r_{i}}^{2}(t)}}$

Here $h_{x, y}$ is the block fading coefficient for the channel between $x$ and $y$ with the channel coherence time be $\omega$ bits.

Relay receives the data stream from both sources and it applies Demodulate and Forward technique on the data stream using Maximum Likelihood (ML) optimum demodulation:

$$
\hat{\mu_{s_{i}}}=\operatorname{argmin}\left\{\left|y_{s_{i} R}-\sqrt{E_{C_{1}}} * h_{s_{i} R} *\left(\mu_{s_{i}}\right)\right|^{2}\right\}
$$

where $\mu$ represents the network-channel coded input symbols generated from $Y_{r_{i}}$

The received coded symbol from the relays at the destination is product of both estimated symbols and represented as: $\hat{\mu_{s 1}} \bigoplus \hat{\mu_{s 2}}$

Network coded signal output at the relay $r_{i}$ for time slot is given as follows:

$$
X_{r_{i}}(t)=\alpha_{i} X_{r_{i}}^{1}(t)+\beta_{i} X_{r_{i}}^{2}(t)
$$

where $i, j \in\{1,2\} .\left\{\alpha_{1}, \alpha_{2}, \beta_{1}, \beta_{2}\right\} \in G F(q)$ is selected such that $\alpha_{1} X_{r_{1}}^{1}(t)+\beta_{1} X_{r_{1}}^{2}(t)$ and $\alpha_{2} X_{r_{2}}^{1}(t)+\beta_{2} X_{r_{2}}^{2}(t)$ are linearly independent.

\section{A. Decoding Phase}

After broadcasting and relaying phase, the destination has a vector of network channel coded sub-symbols $\hat{\mu}_{1}$ and $\hat{\mu}_{2}$ using $\mathbf{y}_{D}=\left[\mathbf{y}_{S_{1} D}, \mathbf{y}_{S_{2} D}, \mathbf{y}_{R_{1} D}, \mathbf{y}_{R_{2} D}\right]$ this information, the destination tries to recover both originally transmitted symbols $S 1$ and $S 2$ transmitted by $(S 1)$ and $(S 2)$ respectively.

The Output of the Linear Block Code at source $s_{i}$ for time slot $t$ :

$$
C_{i}(t)=\left\{c_{i}^{1}(t), c_{i}^{2}(t), \cdots, c_{i}^{M_{i}}(t)\right\}
$$

where $i \in\{1,2\}$.

Since the output of $L-Q A M$ modulator for time slot $t$ at source $s_{i}$ was $Y_{i}(t)$. Without loss of generality we assume that $M$ is multiple of $L$.

And the input signal received from source $s_{i}$ at the relay $r_{j}$ for time slot $t$ was: $Y_{r_{j}}^{i}(t)$, where $i, j \in\{1,2\}$. The Demodulator output for input signal can be formulate as in (10) and (11). $Y_{r_{j}}^{i^{\hat{n}}}(t)$ at the relay $r_{j}$ for time slot $t: X_{r_{i}}^{j}(t)$. Finally the Decoder output for data stream $C_{r}^{i}(t)$ at the relay is given by: $r_{j}$ for time slot $t: X_{r}^{i}(t)$

The estimated network channel coded symbols re-formed at destination using Maximum Likelihood (ML)-Optimum rule can be derive as follows from (12) to (17).

$$
\begin{gathered}
\wedge^{M L}\left(\hat{\mu}_{S}, \hat{\mu}_{R}\right)=\prod_{t=1}^{N_{s}} \exp \left(-\frac{\left|y_{s_{d}}-\sqrt{E_{c}} h_{s_{t} d}\left(\mu_{s_{t}}\right)\right|^{2}}{N_{o}}\right) \\
\times=\prod_{q=1}^{N_{R}}\left[P_{R_{q}}^{(N C)} \exp \left(-\frac{\left|y_{R_{d}}-\sqrt{E_{c}} h_{R_{q} d}\left(\mu_{R_{q}^{(N C)}}\right)\right|^{2}}{N_{o}}\right)\right. \\
\left.\quad+\left(1-P_{R_{q}^{(N C)}}\right) \exp \left(-\frac{\left|y_{R_{d}}-\sqrt{E_{c} i} h_{R_{q} d}\left(\mu_{R_{q}}\right)\right|^{2}}{N_{o}}\right)\right]
\end{gathered}
$$

This is in our case can be modified as:

$$
\hat{\mu}_{s}, \hat{\mu_{R}}=\prod_{t=1}^{2} \exp \left(-\frac{\left|y_{S_{t}}-\sqrt{E_{c}} h_{s_{t} d}\left(\mu_{s_{t}}\right)\right|^{2}}{N_{o}}\right)
$$




$$
\times\left[(1-Q(x))(1-Q(y)) \exp \left(-\frac{\left|y_{R_{d}}-\sqrt{E_{c}} h_{R_{d}}(1-2 \oplus(0,0))\right|^{2}}{N_{o}}\right)\right.
$$$$
+\left[(1-Q(x)) Q(y) \exp \left(-\frac{\left|y_{R_{d}}-\sqrt{E_{c}} h_{R_{d}}(1-2 \oplus(0,1))\right|^{2}}{N_{o}}\right)\right.
$$

$$
+Q(x))(1-Q(y)) \exp \left(-\frac{\left|y_{R_{d}}-\sqrt{E_{c}} h_{R_{d}}(1-2 \oplus(1,0))\right|^{2}}{N_{o}}\right)
$$

$$
+Q(x)) Q(y) \exp \left(-\frac{\left|y_{R_{d}}-\sqrt{E_{c}} h_{R_{d}}(1-2 \oplus(1,1 s))\right|^{2}}{N_{o}}\right)
$$

where

$$
x=\sqrt{\frac{2 E_{R}\left|h_{s_{1} R}\right|^{2}}{N o}}, y=\sqrt{\frac{2 E_{R}\left|h_{s_{2} R}\right|^{2}}{N o}}
$$

Where the estimated symbols from sources and relays are present in the vectors respectively calculation of Average Symbol Error Probability (ASEP). and we have applied the network coding directly on the channel-coded-sub-symbols and it results in higher diversity and low complexity. The proposed collaborative decoder has been shown below at top of page 5 .

\section{Performance Evaluation of Proposed ALGORITHM}

In this section, we analyze the complexity, energy efficiency and error probability trade-off of the proposed scheme. The major considerations are : i) $\sigma_{X Y}^{2}=\sigma^{2}=1 / 2$ for every wireless link; and ii) $E_{S}=E_{c} \log _{2}(M), E_{R_{r}}=E_{c} \log _{2}(M)+$ $E_{c} \log _{2}(N)$ for $r=1,2, \ldots, M$, where $E_{c}$ denotes the average energy per transmitted symbol.

In order to verify our claims about the performance of the proposed scheme, and to compare its performance with two most famous and currently in-use state of the art protocols Decode and Forward and traditional joint network channel coding. The MonteCarlo simulation results have been presented.

Performance evaluation of proposed collaborative scheme is basically composed of two important parameters: 1. Energy Efficiency 2. Error Probability

In Figs. 4-6, the performance of the mathematical framework and algorithm is compared against Monte Carlo simulations for various traditional and state of the art topologies and network codes. The results confirm the good accuracy of the proposed mathematical approach and, more importantly our results substantiate the conclusions about the achievable diversity order and improved energy efficiency while resulting in reduced error probability.

In Fig. 4 numerical result to substantiate the diversity analysis is shown for the expander coding based network coding protocol; the experiment setup was based on $N_{S}=2$, $N_{R}=2, M=16$ and experiment study was conducted on source nodes performance of traditional joint network channel coding scheme with that off expander coding based network coding protocol. The following observations can be made based on results; firstly the sources has diversity order two, that is confirmed by upper- and lower-bounds achieved; secondly reduced error probabilities confirms the robustness of the receiver towards demodulation errors at the relays; and finally the destination is able to reconstruct the symbol streams transmitted from the relays, as confirmed by the error performance curves that show no error-floor and improved energy efficiency as well. Also, to substantiate our proposed approach, result shows the Average Symbol Error Probability (ASEP) computed for both setups. Result clearly show the potential gain of collaborative scheme in the presence of demodulation errors at the relays. Furthermore at ASEP $=10^{-3}$, collaborative scheme allows us to reduce the average energy per transmitted symbol, $E_{c}$, of approximately: $82 \%$ compared with 'traditional joint network channel coding scheme'.

In Fig. 5 numerical results to compare the performance of collaborative scheme with other state-of-the-art relaying protocols are shown. The following relaying protocols are considered for comparison purposes. 1) "Decode-and-forward"in which the relay decodes the source message in one block and transmits the re-encoded message in the other block and 2) "Traditional joint network-channel coding" the experiment setup was the same as explained above. We present the Average Symbol Error Probability (ASEP) performance as a function of the average transmit energy per coded symbol $E c$, and we also investigated the complexity and energy-efficiency trade-off and Relative Average Energy Ratio per transmitted symbol (RAER) has been calculated; it is clear from result that the scheme provides low complex operations and in turn energy efficiency at ASEP $=10^{-3}$ it is possible to achieve energy efficiency as high as up to $97 \%$.

TABLE I

ENERGY EFFICIENCY ANALYSIS (RAER \%).

$\begin{array}{ll}\text { Decode-and-Forward } & 97 \% \\ \text { Joint Network-channel-Coding } & 91 \%\end{array}$

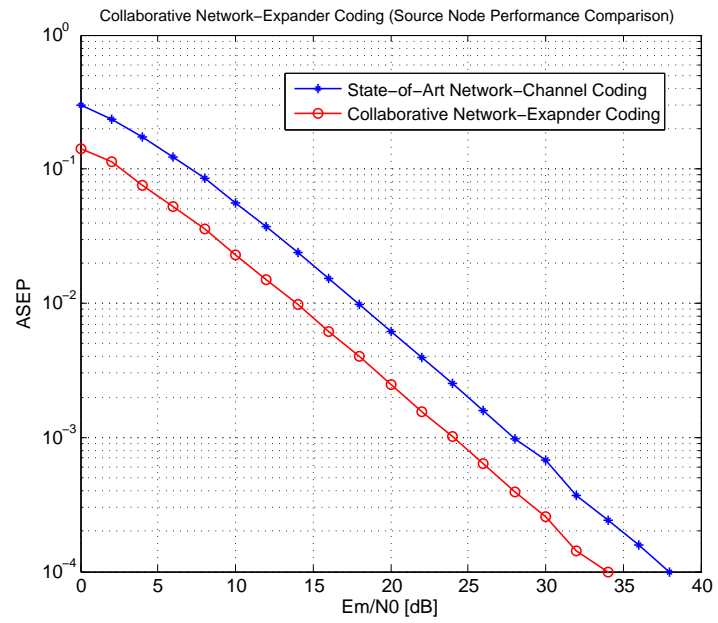

Fig. 4. Expander coding based Network coding protocol. Setup: $N_{S}=2, N_{R}=2$, $M=16$ : Comparison is based on source nodes performance of traditional joint network channel coding scheme with expander coding based network coding protocol 


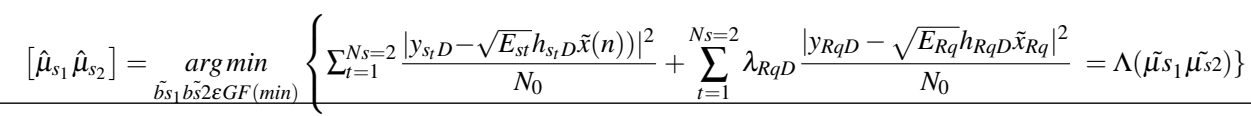

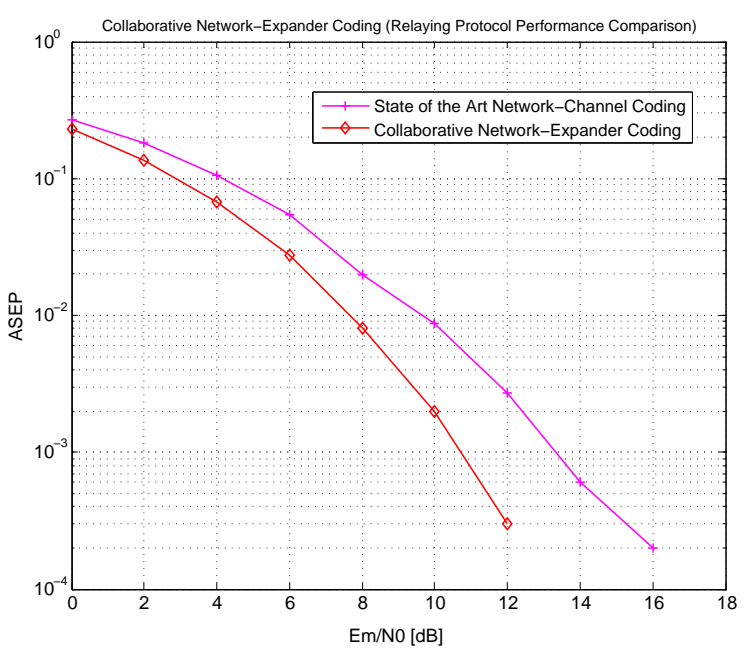

Fig. 5. Expander coding based Network coding protocol. Setup: $N_{S}=2, N_{R}=2$, $M=16$ : Comparison is based on relaying protocol performance of traditional joint network channel coding scheme with expander coding based network coding protocol.

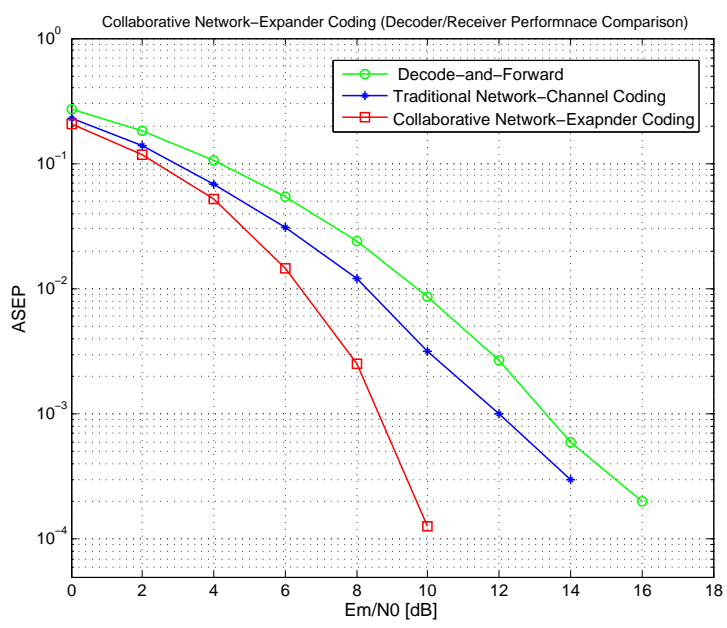

Fig. 6. Expander coding based network coding receiver performance analysis Setup: $N_{S}=2, N_{R}=2, M=16$ : Comparison is based on receiver design performance analysis in comparison with decode-and-forward and traditional joint network channel coding scheme

Fig. 6 shows the simulation results for the same setup using the proposed collaborative decoder. The red curve shows the protocol when the destination uses the presented collaborative expander-network coding receiver as shown in equation. 19. It can be observed that $97 \%$ energy efficiency is achieved with respect to decode-and-forward and in case of traditional joint network-channel coding energy efficiency is enhanced up to $91 \%$.

Table 1 demonstrates the energy efficiency of proposed scheme in comparison with conventional joint networkchannel coding scheme and decode-and-forward for all experiments, the basic purpose of this tables is to provide an quick overview of improved energy efficiency that we have been achieved as an result of proposed algorithm.

\section{CONCLUSION}

In this paper, the performance of collaborative network channel coding based on expander codes has been analyzed in wireless communication network with consideration of practical problems. The presented analytical framework enables reduce symbol error probability and increased energy efficiency and particularly by using the expander codes for channel codes to make polynomial time and simple coding and encoding possible. It provides insightful information about the performance of the system.

\section{ACKNOWLEDGMENT}

This work is supported by the European research project “GREENET" (MITN-GA-2010-264759).

\section{REFERENCES}

[1] R. Ahlswede, N. Cai, S.Y. R. Li, and R. W. Yeung,Network information flow , IEEE Trans. Inform. Theory, vol. 46, no. 4, pp. 12041216, (July 2000).

[2] R. Koetter and F. R. Kschischang, Coding for errors and erasures in random network coding, IEEE Trans. Inform. Theory, vol. 54, no. 8, pp. 35793591, (Aug.2008).

[3] D. Silva, Error control for network coding, Ph.D. Dissertation, University of Toronto, Canada, 2009

[4] J. N. Laneman, D. Tse, and G. Wornell, Cooperative diversity in wireless networks: Efficient protocols and outage behavior, IEEE Trans. Inform. Theory, vol. 50, no. 12, pp. 30623080, (Dec.2004).

[5] Renzo M, Iwaza L, Kieer M, Duhamel P, Agha K: Robust Wireless Network Coding An Overview, Springer Berlin Heidelberg, Volume 45 of Lecture Notes of the Institute for Computer Sciences, Social Informatics and Telecommunications Engineering (2010) chap. 59, :685-698.

[6] N Ahmed, M Khojastepour, B Aazhang, Outage minimization and optimal power control for the fading relay channel. IEEE Information Theory Workshop (ITW)2004 (San Antonio, USA, 2004), pp. 458462

[7] A. Nasri, R. Schober, and M. Uysal, Error rate performance of networkcoded cooperative diversity systems, IEEE Global Commun. Conf., pp. 16 , Dec. 2010.

[8] A. Nasri, R. Schober, and M. Uysal, Performance and optimization of networkcoded cooperative diversity systems, IEEE Trans. Commun., accepted for publication. [Online]. Available: http://www.ece.ubc.ca/amirn/NCCD.pdf

[9] .Guo, J. Huang, B. Wang, S. Zhou, J.-H. Cui, and P. Willett, A practical joint network-channel coding scheme for reliable communication in wireless networks, IEEE Transactions on Wireless Communications, vol. 11, no. 6, pp. 2084 2094, (June 2012).

[10] C. Hausl and P. Dupraz, Joint network-channel coding for the multipleaccess relay channel, Proc. IEEE Commun. Soc. Sensor Ad Hoc Commun. Netw. 3, pp. 817822, 2006.

[11] W. Wesley Peterson and EJ Weldon, 'Error correctin codes of The MIT Press' Revised, 2nd Edition, March 15, 1972; ISBN-10: 0262160390; ISBN-13: 978-0262160391.

[12] S. Zhang, S.C. Liew, P.P. Lam, Hot topic: physical-layer network coding, MobiCom06, Sept. 23-26, 2006, Los Angeles, Calif.

[13] S. Zhang and S.C. Liew, and P.P. Lam, Channel coding and decoding in a relay system operated with physical-layer network coding, IEEE J.Selected Areas in Comm., Vol. 27, No.5, June 2009.

[14] Bletsas A, Khisti A, Reed DP, Lippman A "A Simple Cooperative Diversity Method Based on Network Path Selection", IEEE Journal on Select Areas in Communications, Vol 24(3), pp:659-672 (2006).

[15] Ming X, Aulin T "Optimal Decoding and Performance Analysis of a Noisy Channel Network with Network Coding", IEEE Transactions on Communications, Vol: 57(5) 2009, pp:1402-1412, (2009).

[16] Marium Jalal Chaudhry, Michela Iezzi, Marco Di Renzo, Fabio Graziosi "Joint channel network decoding for the two-source two-relay network", in proc International Workshop on Computer-Aided Modeling Analysis and Design of Communication2012, pp: 100-104. 